\title{
Criticality in the configuration-mixed Interacting Boson Model: (2) $\hat{Q}\left(\chi_{1}\right) \hat{Q}\left(\chi_{1}\right)-\hat{Q}\left(\chi_{2}\right) \hat{Q}\left(\chi_{2}\right)$ mixing
}

\author{
V. Hellemans ${ }^{\text {a,c }}$, P. Van Isacker ${ }^{\text {b }}$, S. De Baerdemacker ${ }^{\mathrm{a}, \mathrm{d}}$, and \\ K. Heyde ${ }^{a}$ \\ ${ }^{a}$ Department of Subatomic and Radiation Physics, Proeftuinstraat 86, B-9000 \\ Gent, Belgium \\ ${ }^{\mathrm{b}}$ Grand Accélérateur National d'Ions Lourds, B.P. 55027, F-14076 Caen Cedex 5, \\ France \\ ${ }^{\mathrm{c}}$ Université Libre de Bruxelles, Service de Physique Nucléaire Théorique, B-1050 \\ Bruxelles, Belgium \\ ${ }^{\mathrm{d}}$ University of Toronto, Department of Physics, Toronto, Ontario M5S 1A\%, \\ Canada
}

\begin{abstract}
The case of $\hat{Q}\left(\chi_{1}\right) \cdot \hat{Q}\left(\chi_{1}\right)-\hat{Q}\left(\chi_{2}\right) \cdot \hat{Q}\left(\chi_{2}\right)$ mixing in the interacting boson model is studied within its mean-field approximation. It is shown that the criticality conditions lead to two classes of solutions and that $\chi_{1}$ and $\chi_{2}$ need to have an opposite sign to allow for prolate-oblate shape coexistence. No evidence for a stable triaxial minimum is found. Phase diagrams for selected cases have been constructed.
\end{abstract}

Key words: Configuration-mixed IBM, phase diagrams PACS: 21.60.Fw, 21.60.Ev, 05.70.Fh, 05.70.Jh

\section{Introduction}

The interacting boson model (IBM) [1] has proven to be tailor-made to study the concept of shape phase transitions in atomic nuclei [2]. Evaluating the expectation value of the Hamiltonian in an intrinsic state, one obtains an analytic expression of the energy surface as a function of the quadrupole shape

Email address: veerle.hellemans@ulb.ac.be (V. Hellemans). 
variables $\beta$ and $\gamma[3-5]$, allowing for a geometrical interpretation of a particular IBM Hamiltonian. For changing parameters in the Hamiltonian, the geometrical picture will alter as well and first- and second-order shape phase transitions have been identified along the transitions from one IBM symmetry limit to another [6-14].

In its most compact form, the basic building blocks of the IBM model are $s$ and $d$ bosons, which carry momentum $J=0$ and $J=2$ respectively. These bosons can be mapped from $J=0$ and $J=2$ valence nucleon pairs, if no distinction is made between proton and neutron bosons. In a subsequent step, which gives rise to the interacting boson model with configuration mixing (IBM-CM) $[15,16]$, the model space can be extended to include particle-hole $(\mathrm{p}-\mathrm{h})$ excitations across a closed proton or neutron shell. These $\mathrm{p}-\mathrm{h}$ excitations can descend very low in energy in certain regions of the nuclear chart $[17,18]$ and can mix significantly with the regular configuration (which contains only valence bosons). From a macroscopic point of view, this effect can be associated with the occurrence of several minima in the energy surface within a relatively small energy range, a phenomenon named shape coexistence. In the case of the IBM-CM, the energy surface is constructed by evaluating the expectation value of the Hamiltonian in an appropriate intrinsic state [19]. The analytical form of the energy surface allows us to examine for which range of the parameters an energy surface results with more than one minimum. Hence, the relation between the microscopic phenomenon of configuration mixing and the macroscopic occurrence of several coexisting minima in the energy surface can be studied.

In a preceding paper [20], we have explored the energy surface associated with the mixing between quadrupole vibrational motion, characterised by the $U(5)$ limit of the IBM, and the $\gamma$-independent and axially symmetric rotational motion, both contained in the quadrupole interaction. It was demonstrated that a large region of shape coexistence between a spherical and a deformed minimum is found for a broad range of excitation energies of the intruder $2 \mathrm{p}-2 \mathrm{~h}$ configuration. This is not the case for the IBM without configuration mixing, where the energy surface only exhibits two shallow coexisting minima for a small range of parameters. Moreover, a first-order shape phase transition occurs when passing through the regions of shape coexistence. In the case of $U(5)-O(6)$ mixing, the deformation of the global minimum exhibits a powerlaw behaviour at the critical point in the transition from a spherical to a deformed minimum. Hence, this transition is identified as of second order.

It is, however, equally important to study mixing between oblate-oblate, prolateprolate, and oblate-prolate deformed configurations, as well as mixing resulting when $\gamma$-independent and prolate or oblate deformed minima appear. The Pt and the Os region provide a good example to study the latter possibilities. In a very recent paper [21], the geometry of the Pt isotopes was studied, showing 
the evolution from a spherical to an oblate and finally to a prolate shape in the ground state, with the occurrence of shape coexistence in ${ }^{182,184,186,188} \mathrm{Pt}$. In this article, we will present a study of the evolving geometry of mixing between two deformed configurations. This encompasses various mixing situations between the different IBM dynamical symmetry limits, i.e. the $S U(3)$, the $\overline{S U}(3)$ and the $O(6)$ limit. The $S U(3)(\overline{S U}(3))$ dynamical symmetry limit is associated with a vibrating axially symmetric prolate (oblate) rotor. Similarly the $O(6)$ limit corresponds to a $\gamma$ independent rotor.

Studies of the energy surface associated with the IBM Hamiltonian without configuration mixing have shown that the energy surface passes through a critical point at the $O(6)$ limit in the transition from $S U(3)$ to $\overline{S U}(3)$ [9]. A detailed analysis of the energy surface [7] in the full parameter space of the IBM without configuration mixing has proven that only shape coexistence between a spherical and a deformed minimum can be found. So, shape coexistence between a prolate and oblate minimum is absent in this case.

In section 2, we succinctly introduce the IBM Hamiltonian for configuration mixing and the energy surface that can be associated with it. In section 3, the necessary ingredients for the study of the qualitative behaviour of the energy surfaces as a function of the IBM parameters are briefly recapitulated. Due to the complexity of the energy surface, only limited information can be extracted analytically. Analytical results are presented in sections 3 and 4 . Finally, phase diagrams are presented for selected cases in section 5 .

\section{The energy surface for $\hat{Q}\left(\chi_{1}\right) \hat{Q}\left(\chi_{1}\right)-\hat{Q}\left(\chi_{2}\right) \hat{Q}\left(\chi_{2}\right)$ mixing}

In the following, we briefly summarise the ideas and equations essential for the construction of the energy surface. For an extensive introduction to the IBM and its mean-field interpretation, we refer the reader to [1,22]. A commonly used form of the IBM Hamiltonian, which captures the essential features of the model, is the so-called consistent-Q Hamiltonian [23]

$$
\hat{H}_{\mathrm{cqf}}=\epsilon \hat{n}_{d}-|\kappa| \hat{Q}(\chi) \cdot \hat{Q}(\chi)
$$

The operator $\hat{n}_{d}$ is the $d$-boson number operator and $\hat{Q}_{\mu}(\chi)$ is the quadrupole operator. For specific choices of the parameters, analytically solvable symmetry limits result: (1) the $U(5)$ limit for $\kappa=0,(2)$ the $O(6)$ limit for $(\varepsilon=0, \chi=$ $0)$, (3) the $S U(3)$ symmetry limit for $(\varepsilon=0, \chi=-\sqrt{7} / 2)$ and (4) the $\overline{S U}(3)$ symmetry limit for $(\varepsilon=0, \chi=\sqrt{7} / 2)$. Calculation of the expectation value of the Hamiltonian in a normalised projective coherent state or intrinsic state 


$$
|N, \beta, \gamma\rangle=\frac{1}{\sqrt{N !}\left(1+\beta^{2}\right)^{N / 2}}\left(s^{\dagger}+\beta\left[\cos \gamma d_{0}^{\dagger}+\frac{1}{\sqrt{2}} \sin \gamma\left(d_{2}^{\dagger}+d_{-2}^{\dagger}\right)\right]\right)^{N}|0\rangle
$$

results in an energy surface depending on the collective shape variables $(\beta, \gamma)$,

$$
\begin{aligned}
E^{N}(\epsilon,|\kappa|, \chi, \beta, \gamma) & \equiv\left\langle N, \beta, \gamma\left|\hat{H}_{\mathrm{cqf}}\right| N, \beta, \gamma\right\rangle \\
& =\epsilon N \frac{\beta^{2}}{1+\beta^{2}}-|\kappa|\left[\frac{N\left[5+\left(1+\chi^{2}\right) \beta^{2}\right]}{1+\beta^{2}}\right. \\
& \left.+\frac{N(N-1)}{\left(1+\beta^{2}\right)^{2}}\left(\frac{2}{7} \chi^{2} \beta^{4}-4 \sqrt{\frac{2}{7}} \chi \beta^{3} \cos (3 \gamma)+4 \beta^{2}\right)\right]
\end{aligned}
$$

where $N$ denotes the number of valence bosons. In the $U(5)$ limit, the energy surface exhibits a spherical minimum, the $O(6)$ limit corresponds to an energy surface with a $\gamma$-independent deformed minimum and in the $S U(3)$ limit, the energy surface exhibits a prolate deformed minimum for $\chi=-\sqrt{7} / 2$ (denoted $S U(3)$ ) and an oblate deformed minimum for $\chi=+\sqrt{7} / 2$ (indicated with $\overline{S U}(3))$.

The interacting boson model with configuration mixing (IBM-CM) $[15,16]$ allows for the description of intruder states, i.e. low-lying particle-hole excitations in the energy spectrum. Within the IBM-CM framework $2 \mathrm{p}-2 \mathrm{~h}, 4 \mathrm{p}-4 \mathrm{~h}, \ldots$ excitations are described as $N+2, N+4, \ldots$ boson configurations, respectively. The Hamiltonian for the description of configuration mixing between regular valence states and the intruder $2 \mathrm{p}-2 \mathrm{~h}$ states is written as

$$
\hat{H}=\hat{P}_{N}^{\dagger} \hat{H}_{\mathrm{cqf}}^{N} \hat{P}_{N}+\hat{P}_{N+2}^{\dagger}\left(\hat{H}_{\mathrm{cqf}}^{N+2}+\Delta\right) \hat{P}_{N+2}+\hat{V}_{\mathrm{mix}}
$$

where $\hat{P}_{N}$ and $\hat{P}_{N+2}$ are operators projecting onto the $[N]$ and $[N+2]$ boson spaces, respectively. The different superscripts in $\hat{H}_{\text {cqf }}^{N}$ and $\hat{H}_{\text {cqf }}^{N+2}$ indicate that the parametrisation can be configuration-dependent. The parameter $\Delta$ is associated with the energy needed to excite two particles across a shell gap, corrected for the pairing interaction and a monopole effect [24]. Finally, the interaction between the regular and the intruder configuration is described by $\hat{V}_{\text {mix }} \equiv w_{0}\left(s^{\dagger} s^{\dagger}+s s\right)+w_{2}\left(d^{\dagger} \cdot d^{\dagger}+\tilde{d} \cdot \tilde{d}\right)$. By means of a matrix coherent state method [19], one obtains the geometric interpretation of the IBM-CM. The energy surface in $(\beta, \gamma)$ associated with the Hamiltonian (4) is given by the lowest eigenvalue of the matrix

$$
\left[\begin{array}{cc}
E^{N}\left(\epsilon_{1},\left|\kappa_{1}\right|, \chi_{1}, \beta, \gamma\right) & \omega(\beta) \\
\omega(\beta) & E^{N+2}\left(\epsilon_{2},\left|\kappa_{2}\right|, \chi_{2}, \beta, \gamma\right)+\Delta
\end{array}\right]
$$


with

$$
\omega(\beta) \equiv\left\langle N, \beta, \gamma\left|\hat{V}_{\text {mix }}\right| N+2, \beta, \gamma\right\rangle=\sqrt{(N+2)(N+1)} \frac{w_{0}+w_{2} \beta^{2}}{1+\beta^{2}}
$$

and $E^{N}\left(\epsilon_{1},\left|\kappa_{1}\right|, \chi_{1}, \beta, \gamma\right)$ and $E^{N+2}\left(\epsilon_{2},\left|\kappa_{2}\right|, \chi_{2}, \beta, \gamma\right)$ the expectation values of $\hat{H}_{\mathrm{cqf}}^{N}$ and $\hat{H}_{\mathrm{cqf}}^{N+2}$ in the appropriate intrinsic state. The choice $w_{0}=w_{2} \equiv w$ leads to a $\beta$-independent $\omega$.

In the present paper, we focus our interest on configuration mixing between pure rotational regular and intruder configurations. The energy surface for $\hat{Q}\left(\chi_{1}\right) \cdot \hat{Q}\left(\chi_{1}\right)-\hat{Q}\left(\chi_{2}\right) \cdot \hat{Q}\left(\chi_{2}\right)$ mixing obtained with the matrix coherent state method is written as

$$
\begin{aligned}
& E_{-}=\frac{|\kappa|}{2\left(1+\beta^{2}\right)^{2}}\left[a_{1} \beta^{4}+a_{2} \beta^{2}+a_{3} \beta^{3} \cos (3 \gamma)+a_{4}\right. \\
& \left.\quad-\left[\left(b_{1} \beta^{4}+b_{2} \beta^{2}+b_{3} \beta^{3} \cos (3 \gamma)+b_{4}\right)^{2}+\omega^{\prime 2}\left(1+\beta^{2}\right)^{4}\right]^{1 / 2}\right],
\end{aligned}
$$

with

$$
\begin{aligned}
& a_{1}=-N\left(1+\frac{1}{7}(2 N+5) \chi_{1}^{2}\right)-\left|\sigma^{\prime}\right|(N+2)\left(1+\frac{1}{7}(2 N+9) \chi_{2}^{2}\right)+\Delta^{\prime}, \\
& a_{2}=-N\left(\chi_{1}^{2}+2(2 N+1)\right)-\left|\sigma^{\prime}\right|(N+2)\left(\chi_{2}^{2}+2(2 N+5)\right)+2 \Delta^{\prime}, \\
& a_{3}=\frac{4}{7} \sqrt{14}\left(N(N-1) \chi_{1}+\left|\sigma^{\prime}\right|(N+1)(N+2) \chi_{2}\right), \\
& a_{4}=-5\left(N+\left|\sigma^{\prime}\right|(N+2)\right)+\Delta^{\prime}, \\
& b_{1}=-N\left(1+\frac{1}{7}(2 N+5) \chi_{1}^{2}\right)+\left|\sigma^{\prime}\right|(N+2)\left(1+\frac{1}{7}(2 N+9) \chi_{2}^{2}\right)-\Delta^{\prime}, \\
& b_{2}=-N\left(\chi_{1}^{2}+2(2 N+1)\right)+\left|\sigma^{\prime}\right|(N+2)\left(\chi_{2}^{2}+2(2 N+5)\right)-2 \Delta^{\prime}, \\
& b_{3}=\frac{4}{7} \sqrt{14}\left(N(N-1) \chi_{1}-\left|\sigma^{\prime}\right|(N+1)(N+2) \chi_{2}\right), \\
& b_{4}=-5\left(N-\left|\sigma^{\prime}\right|(N+2)\right)-\Delta^{\prime} .
\end{aligned}
$$

We defined $\left|\sigma^{\prime}\right| \equiv|\sigma| /|\kappa|, \Delta^{\prime} \equiv \Delta /|\kappa|$ and $\omega^{\prime} \equiv 2 \omega /|\kappa|$ and made a distinction between the notation for the quadrupole strength in the configuration containing $N$ bosons, which we call $\kappa$, and the quadrupole strength for the configuration with $N+2$ bosons, which is denoted with $\sigma$. The factor $|\kappa| / 2$ in (7) will be omitted from now on as it is a scaling factor without influence on the structural properties of the energy surface. 


\section{The criticality conditions}

Aiming to explore for which parameters $\left(\left|\sigma^{\prime}\right|, \chi_{1}, \chi_{2}, \Delta^{\prime}, \omega^{\prime}, N\right)$ in the full parameter space several minima coexist in the energy surface $(7)$, we need a mathematical tool to study the qualitative behaviour of a family of energy surfaces $E_{-}\left(\beta, \gamma ;\left|\sigma^{\prime}\right|, \chi_{1}, \chi_{2}, \Delta^{\prime}, \omega^{\prime}, N\right)$. Such a study can be performed using the ideas of catastrophe theory, which we will now succinctly describe. We refer the reader to [25] and [20] for more elaborate discussions. In the language of catastrophe theory, the parameters $\left(\left|\sigma^{\prime}\right|, \chi_{1}, \chi_{2}, \Delta^{\prime}, \omega^{\prime}, N\right)$ in (7)-(8) are referred to as the control parameters, whereas $(\beta, \gamma)$ are the quadrupole shape variables. Changes in the qualitative behaviour of the energy surface are heralded by the occurrence of degenerate critical points. These points are determined through the criticality conditions

$$
\frac{\partial E_{-}}{\partial \beta}=0, \quad \frac{\partial E_{-}}{\partial \gamma}=0, \quad \operatorname{det}(\mathcal{S})=0,
$$

where the stability matrix $\mathcal{S}$ is defined as

$$
\mathcal{S} \equiv\left(\begin{array}{cc}
\frac{\partial^{2} E_{-}}{\partial \beta^{2}} & \frac{\partial^{2} E_{-}}{\partial \beta \partial \gamma} \\
\frac{\partial^{2} E_{-}}{\partial \gamma \partial \beta} & \frac{\partial^{2} E_{-}}{\partial \gamma^{2}}
\end{array}\right) .
$$

These conditions define relations between the control parameters in the IBMCM Hamiltonian $\left(\left|\sigma^{\prime}\right|, \chi_{1}, \chi_{2}, \Delta^{\prime}, \omega^{\prime}, N\right)$. When fulfilled, the energy surface in $(\beta, \gamma)$ exhibits a degenerate critical point. Hence, they define the locus of points in control parameter space which separates the regions in which the qualitative properties of the energy surface remain unchanged.

In the preceeding [20], we studied the energy surface associated with $U(5)-$ $\hat{Q}(\chi) \cdot \hat{Q}(\chi)$ configuration mixing. In that case, an analytical solution to the criticality conditions could be derived by expanding the energy surface around the point $(\beta, \gamma)=(0, n \pi / 3)$. The degenerate critical points thus obtained indicated a transition from a spherical to a deformed minimum in the energy surface. For the energy surface we study at present, it is not possible to deduce an interesting analytical solution to the set of equations (9) by expanding the energy surface around the point $(\beta, \gamma)=(0, n \pi / 3)$. It can be shown that the only meaningful solution occurs for the number of bosons $N<2$. This is not too surprising given the fact that we mix unperturbed energy surfaces with deformed minima. Hence, structural changes in the energy surface are unlikely to occur at $\beta=0$.

Since no significant analytical solution can be obtained through a Taylor expansion, we have to solve the criticality conditions (9) numerically. In the following, we will show that the physically interesting solutions can be categorised into two classes, which allows us to draw some general conclusions. 
Furthermore, this simplifies the numerical calculation of the critical points significantly. Calculating the first derivative of the energy surface with respect to $\gamma, \frac{\partial E_{-}}{\partial \gamma}=0$, we can single out the term $\beta^{3} \sin (3 \gamma)$, which results in the expression (see Appendix A)

$$
\frac{\partial E_{-}}{\partial \gamma}=\frac{3 \beta^{3} \sin (3 \gamma)}{\left(1+\beta^{2}\right)^{2}} F\left(\beta, \gamma ;\left|\sigma^{\prime}\right|, N, \Delta^{\prime}, \chi_{1}, \chi_{2}, \omega^{\prime}\right) .
$$

For the choice $\beta=0$, the solution to the remaining criticality conditions in (9) is the same as the analytical solution obtained from solving the criticality conditions following from a Taylor expansion in $(\beta, \gamma)=(0, n \pi / 3)$, which was not meaningful. However, if we consider $\gamma$ equal to $n \pi / 3$, the criticality conditions (9) reduce to

$$
\left.\frac{\partial E_{-}}{\partial \beta}\right|_{\gamma=n \pi / 3}=0, \quad \operatorname{det}\left(\begin{array}{cc}
\left.\frac{\partial^{2} E_{-}}{\partial \beta^{2}}\right|_{\gamma=n \pi / 3} & 0 \\
0 & \left.\frac{\partial^{2} E_{-}}{\partial \gamma^{2}}\right|_{\gamma=n \pi / 3}
\end{array}\right)=0 .
$$

We can distinguish two classes of solutions. In a first class, the degenerate critical points are obtained from the conditions

$$
\left.\frac{\partial E_{-}}{\partial \beta}\right|_{\gamma=n \pi / 3}=0 \quad \text { and }\left.\quad \frac{\partial^{2} E_{-}}{\partial \beta^{2}}\right|_{\gamma=n \pi / 3}=0 \text {. }
$$

The locus of degenerate critical points obtained as a solution to these conditions indicates changes in the number of extrema in the $\beta$-direction (while $\gamma=n \pi / 3)$. Alternatively, a second class of solutions to

$$
\left.\frac{\partial E_{-}}{\partial \beta}\right|_{\gamma=n \pi / 3}=0 \quad \text { and }\left.\quad \frac{\partial^{2} E_{-}}{\partial \gamma^{2}}\right|_{\gamma=n \pi / 3}=0 \text {. }
$$

determines the degenerate critical points which delineate regions with different structural behaviour in $\gamma$. In fact, it can be shown that shape coexistence between a prolate and an oblate minimum can only arise if $\chi_{1}$ and $\chi_{2}$ have an opposite sign. Indeed, if $a_{3} \neq 0$, the constraint $\left.\frac{\partial^{2} E_{-}}{\partial \gamma^{2}}\right|_{\gamma=n \pi / 3}=0$ (see Appendix A) gives rise to the relation

$$
\omega^{\prime}= \pm \sqrt{\left(b_{3}-a_{3}\right)\left(b_{3}+a_{3}\right)} \frac{b_{1} \beta^{4}+b_{2} \beta^{2}+b_{3} \beta^{3}+b_{4}}{a_{3}\left(1+\beta^{2}\right)^{2}} .
$$

The factor $\sqrt{\left(b_{3}-a_{3}\right)\left(b_{3}+a_{3}\right)}$ determines whether $\omega^{\prime}$ is real or imaginary. Since

$$
\sqrt{\left(b_{3}-a_{3}\right)\left(b_{3}+a_{3}\right)}=\frac{8}{7} \sqrt{-14\left|\sigma^{\prime}\right|(N-1) N(N+1)(N+2) \chi_{1} \chi_{2}},
$$

the solution (15) for $\omega^{\prime}$ derived from the constraint $\partial^{2} E_{-} / \partial \gamma^{2}=0$ is only real or, otherwise stated, shape coexistence between a prolate and an oblate 
minimum is in principle only possible if $\chi_{1}$ and $\chi_{2}$ have opposite sign. Note that eq. (15) is not a solution to the criticality conditions (14) but only a solution

to the constraint $\left.\frac{\partial^{2} E_{-}}{\partial \gamma^{2}}\right|_{\gamma=n \pi / 3}=0$. If $a_{3}$ equals zero, $\chi_{1}$ and $\chi_{2}$ automatically have an opposite sign.

In addition to the previous solutions, another class may arise from the condition $F\left(\beta, \gamma ;\left|\sigma^{\prime}\right|, N, \Delta^{\prime}, \chi_{1}, \chi_{2}, \omega^{\prime}\right)=0$ (see eq. (11)). However, it can easily be seen from the results in Appendix A that the critical point determined by $F\left(\beta, \gamma ;\left|\sigma^{\prime}\right|, N, \Delta^{\prime}, \chi_{1}, \chi_{2}, \omega^{\prime}\right)=0$ is always a maximum in the $\gamma$-direction, and hence this solution is of no interest for our analysis. Nevertheless, it has important consequences since this implies the absence of triaxial minima for any set of control parameters in the case of $\hat{Q}\left(\chi_{1}\right) \hat{Q}\left(\chi_{1}\right)-\hat{Q}\left(\chi_{2}\right) \hat{Q}\left(\chi_{2}\right)$ mixing. Triaxiality is also absent in the IBM without configuration mixing. If a distinction between proton and neutron bosons is made (IBM-2), triaxial phases are possible [26]. Also, the inclusion of higher order interactions [27] or the presence of hexadecupole nucleon pairs [28] gives rise to triaxiality.

\section{Behaviour of the energy surface near a degenerate critical point}

Before we determine the phase diagrams for various mixing cases, it is of interest to study the behaviour of the energy surface at the degenerate critical points by means of the low-order terms in the Taylor expansion. If we assume that $\beta_{0}$ is the solution of the condition $\partial E_{-} / \partial \beta=0$, the Taylor expansion around the point $(\beta, \gamma)=\left(\beta_{0}, 0\right)$ can formally be written as

$$
\begin{aligned}
E_{-}= & t_{00}+t_{20}\left(\beta-\beta_{0}\right)^{2}+t_{02} \gamma^{2}+t_{30}\left(\beta-\beta_{0}\right)^{3}+t_{12}\left(\beta-\beta_{0}\right) \gamma^{2} \\
& +t_{40}\left(\beta-\beta_{0}\right)^{4}+t_{22}\left(\beta-\beta_{0}\right)^{2} \gamma^{2}+t_{04} \gamma^{4}+\ldots,
\end{aligned}
$$

in which the linear terms are zero. The cross terms $\gamma\left(\beta-\beta_{0}\right), \gamma\left(\beta-\beta_{0}\right)^{2}$, $\gamma\left(\beta-\beta_{0}\right)^{3}$ and $\gamma^{3}\left(\beta-\beta_{0}\right)$, and the term in $\gamma^{3}$ vanish because of the cosine dependence of the energy surface (7) on $\gamma$ and the choice $\gamma=0$.

In a degenerate critical point of the first class (solution to (13)), the coefficient $t_{20}$ vanishes. Hence, up to the cubic terms and neglecting the constant factor $t_{00}$, the energy surface near the critical point can be written as

$$
E_{-} \simeq t_{02} \gamma^{2}+t_{30}\left(\beta-\beta_{0}\right)^{3}+t_{12}\left(\beta-\beta_{0}\right) \gamma^{2}
$$

Consequently, the energy surface exhibits a $\left(\beta-\beta_{0}\right)^{3}$ behaviour at the critical point in its evolution from an energy surface with one deformed minimum in the $\beta$-direction to a surface with two deformed minima in the $\beta$-direction. This process is schematically illustrated in Fig. 1. A similar $\beta^{3}$ behaviour is found at the degenerate critical points along the transition from a deformed minimum to shape coexistence of two minima in the case of $U(5)-S U(3)$ mixing [20]. 
The behaviour of the energy surface at a degenerate critical point of the second class is derived in an analogous way. Because the condition (14) implies that $t_{02}$ in eq. (17) vanishes, the Taylor expansion at the critical point up to quartic order becomes

$$
\begin{gathered}
E_{-} \simeq t_{20}\left(\beta-\beta_{0}\right)^{2}+t_{30}\left(\beta-\beta_{0}\right)^{3}+t_{12}\left(\beta-\beta_{0}\right) \gamma^{2} \\
+t_{40}\left(\beta-\beta_{0}\right)^{4}+t_{22}\left(\beta-\beta_{0}\right)^{2} \gamma^{2}+t_{04} \gamma^{4}
\end{gathered}
$$

where the constant $t_{00}$ is neglected because the overall properties of the energy surface are independent of this term. The $\gamma^{4}$ behaviour of the energy surface at the degenerate critical point of the second class (14) is formally the same as the behaviour in $\beta$ at the triple point along the transition from $U(5)$ to $O(6)$ in the IBM without configuration mixing [8]. The same is true for the energy surface at the degenerate critical points in the spherical-deformed transition in the case of $U(5)-S U(3)$ mixing [20]. Note that the $\gamma^{4}$ character of the germ, combined with the knowledge that no triaxial minima can occur, completely determines the qualitative behaviour of the energy surface at and in the vicinity of the critical point. A schematic example of the evolution in the neighbourhood of the degenerate critical point is shown in Fig. 2.

\section{$5 \quad$ Phase diagrams for $\hat{Q}\left(\chi_{1}\right) \cdot \hat{Q}\left(\chi_{1}\right)-\hat{Q}\left(\chi_{2}\right) \cdot \hat{Q}\left(\chi_{2}\right)$ mixing}

In section 3, we have shown that two classes of degenerate critical points exist. One class indicates critical changes in the number of extrema in the $\beta$ direction, while the other class marks out regions with different behaviour in $\gamma$. This second class of degenerate critical points only appears for opposite signs of $\chi_{1}$ and $\chi_{2}$. Since the full control parameter phase space $\left(N,\left|\sigma^{\prime}\right|, \Delta^{\prime}, \omega^{\prime}, \chi_{1}, \chi_{2}\right)$ turns out too large to handle, we concentrate on the selected cases of $S U(3)$ $\hat{Q}\left(\chi_{2}\right) \cdot \hat{Q}\left(\chi_{2}\right)$ mixing and $O(6)-O(6)$ mixing. For $S U(3)-\hat{Q}\left(\chi_{2}\right) \cdot \hat{Q}\left(\chi_{2}\right)$ mixing, phase diagrams are presented for several values of $\chi_{2}$, providing an overview of the evolution of the phase diagram when the energy surface associated with the intruder configuration evolves from prolate $\left(\chi_{2}=-\sqrt{7} / 2\right)$ to oblate $\left(\chi_{2}=\sqrt{7} / 2\right)$. Unless differently stated, the phase diagrams are calculated for boson number $N=10$. In our numerical calculations, we chose the value of $\gamma$ zero.

\subsection{Phase diagrams for $S U(3)-\hat{Q}\left(\chi_{2}\right) \cdot \hat{Q}\left(\chi_{2}\right)$}

\subsection{1 $S U(3)-S U(3)$ mixing $\left(\chi_{1}=-\sqrt{7} / 2\right.$ and $\left.\chi_{2}=-\sqrt{7} / 2\right)$}

In Sect. 3, we pointed out that degenerate points of the second class (see eq. (14)) only appear whenever $\chi_{1}$ and $\chi_{2}$ have opposite signs. Consequently, when 
configuration mixing between two $S U(3)\left(\chi_{1}=\chi_{2}=-\sqrt{7} / 2\right)$ configurations is considered, we may leave this class out of consideration and focus on the first class (see eq. (13)). It turns out that no (numerical) solutions to these criticality conditions are found in the particular case of $N=10$ bosons. Hence, no critical changes occur in the $\beta$-direction in the intersection $\left(\left|\sigma^{\prime}\right|, \omega^{\prime}\right)$ of the phase space for $\left(\chi_{1}=\chi_{2}=-\sqrt{7} / 2\right)$ and $N=10$. The resulting energy surface always exhibits one deformed minimum. The absence of a region with shape coexistence can intuitively be understood by comparing the deformation of the minima of the energy surfaces in the unperturbed configurations. In panel (a) of Fig. 3, we display the evolution of the ratio $\beta_{N} / \beta_{N+2}$ when the number of bosons $N$ is varied ( $\beta_{N}$ and $\beta_{N+2}$ denote the deformation of the minima in the unperturbed regular and intruder configurations, respectively). This ratio depends on $\chi_{1}, \chi_{2}$ and the boson number $N$, and converges rather quickly to one if $\chi_{1}=\chi_{2}=-\sqrt{7} / 2$. For $N=10$, we do not find a solution to the criticality conditions because the minima of the energy surface associated with the unperturbed regular and intruder configuration occur at approximately the same deformation. Then, intuitively, we do not expect two separated minima in the energy surface resulting from configuration mixing. This argument implies that shape coexistence will be more likely to arise if the ratio $\beta_{N} / \beta_{N+2}$ differs sufficiently from one. We illustrate this idea with the phase diagram for $N=2$ and $\Delta^{\prime}=0$ in panel (b) of Fig. 3. For $N=2$, the ratio $\beta_{N} / \beta_{N+2}$ differs substantially from 1 and we find a very small region of shape coexistence of two prolate deformed minima. The inset contour plots in the $(\beta, \gamma)$ plane and the plot with the energy surface as a function of $\beta$ for $\gamma=0$, however, illustrate that the minima are very shallow. Whereas two distinct minima are present from a mathematical point of view, the barrier between the two minima seems too small from a physics point of view to expect shape coexistence effects to appear. Note that projection onto angular momentum is needed in order to draw a definite conclusion on the importance of the energy barrier between the two minima. We also point out that the degenerate critical points which mark the triangular region of shape coexistence in Fig. 3, are solutions to the criticality conditions of the first class for $\beta>0$. A similar solution is found for $\beta<0$. Because the latter solutions indicate shape coexistence of oblate saddle points (see Appendix B for a proof), we have not displayed these degenerate critical points in the phase diagram.

\subsubsection{Prolate-prolate mixing $\left(\chi_{1}=-\sqrt{7} / 2, \chi_{2}=-\sqrt{7} / 4\right.$ and $\left.\chi_{2}=-\sqrt{7} / 16\right)$}

In Fig. 4, we display similar phase diagrams for mixing between the $S U(3)$ limit and a quadrupole interaction with $\chi_{2}=-\sqrt{7} / 4$ (panel (a)) and $\chi_{2}=$ $-\sqrt{7} / 16$ (panel $(\mathrm{b})$ ), both for $\Delta^{\prime}=0$. In both cases a triangular region of shape coexistence marked out by degenerate critical points of the first class (see eq. (13)) is present, embedded in a region where the energy surface has a deformed prolate minimum. The area of shape coexistence enlarges when $\chi_{2}$ 
evolves further away from its value in the $S U(3)$ limit $\left(\chi_{2}=-\sqrt{7} / 2\right)$. Similar to the results for mixing between two pure $S U(3)$ limits in Fig. 3, the inset $(\beta, \gamma)$ contour plot and the plot with the energy surface as a function of $\beta$ for $\gamma=0$ illustrate that the two minima are rather shallow. In panel (b), it can be seen from the contour plot with shape coexistence that one of the minima is rather soft in the $\gamma$-direction. This is due to the small value of $\chi_{2}$. Indeed, if the value $\chi=0$ is used in the quadrupole interaction, we recover the $O(6)$ limit which is associated with a $\gamma$-independent rotor.

No structural changes occur in the phase diagram if the scaled excitation energy of the intruder states $\Delta^{\prime}$ increases. For increasing $\Delta^{\prime}$, the 'triangle' with shape coexistence shifts to higher values of $\left|\sigma^{\prime}\right|$ and becomes slightly tilted, but no additional loci of degenerate critical points occur in the diagram.

\subsection{3 $S U(3)-O(6)$ mixing $\left(\chi_{1}=-\sqrt{7} / 2\right.$ and $\left.\chi_{2}=0\right)$}

The evolution of the phase diagram for configuration mixing between the dynamical symmetry limits $S U(3)$ and $O(6)$ for various excitation energies of the intruder configuration $O(6)$ is displayed in Fig. 5. If we substitute $\chi_{2}=0$ in eq. (15), we find that the constraint $\partial^{2} E_{-} / \partial \gamma^{2}=0$ implies the value $\omega^{\prime}=0$. Hence, degenerate critical points of the second class (14) only occur for $\omega^{\prime}=0$ which is of no interest. The degenerate critical points displayed in Fig. 5 fulfil the condition (13) and separate regions with a different number of extrema in the $\beta$-direction. A triangular region with shape coexistence between a prolate minimum and an almost $\gamma$-independent prolate minimum is found for all $\Delta^{\prime}$. Again, the two minima are rather shallow. As $\Delta^{\prime}$ increases, the area with shape coexistence shifts to higher values of $\sigma^{\prime}$.

Outside the triangular region of shape coexistence, the energy surface exhibits a deformed prolate minimum. We display the two extremal variants of the energy surface with a deformed prolate minimum in each panel of Fig. 5. Although the energy surface mathematically never becomes $\gamma$-independent at the minimum, the inset contour plots demonstrate that the slope in the $\gamma$ direction may become so flat that we can consider it as such. In general, the behaviour in $\gamma$ evolves from a distinct prolate minimum over intermediate cases to an almost $\gamma$-independent case. Unfortunately, the criticality conditions do not distinguish between those physically different energy surfaces. Therefore, we displayed the two extremal variants of the energy surface, although their position in the diagram is not known a priori. 


\subsubsection{Prolate-oblate mixing $\left(\chi_{1}=-\sqrt{7} / 2\right.$ and $\left.\chi_{2}=\sqrt{7} / 16\right)$}

The phase diagrams for mixing between a prolate and an oblate configuration $\left(\chi_{1}=-\sqrt{7} / 2\right.$ and $\left.\chi_{2}=\sqrt{7} / 16\right)$ are presented in Fig. 6 for different values of $\Delta^{\prime}$. We emphasise that the scale of the $\left|\sigma^{\prime}\right|$ and the $\omega^{\prime}$ axes changes significantly. In addition to the first class of degenerate critical points (13) (indicated in blue) which was extensively discussed in the previous Sect. 5.1.1-5.1.3, a second class (solution to (14) and displayed in black), which announces critical changes in the $\gamma$-direction, arises now that $\chi_{1}$ and $\chi_{2}$ have an opposite sign. The inset $(\beta, \gamma)$ contour plots illustrate the structure of the energy surface changes for each region in the phase diagram. At the left side of the critical points of the second class (black lines in Fig. 6), the energy surface exhibits a deformed prolate minimum. Between the two lines, a second local oblate minimum is created and shape coexistence between a prolate and an oblate minimum takes place. This oblate minimum is rather soft in the $\gamma$-direction due to the small value of $\chi_{2}$. With increasing $\left|\sigma^{\prime}\right|$, the deformed oblate minimum lowers in energy and becomes the global minimum of the energy surface. Finally, for higher values of $\left|\sigma^{\prime}\right|$, the prolate deformed minimum disappears but the oblate minimum remains. The different phase diagrams demonstrate the shift of the region with prolate-oblate shape coexistence to higher ratios of the strengths of the quadrupole interaction $\left|\sigma^{\prime}\right|$ with increasing $\Delta^{\prime}$. Furthermore, the slope of the sets of degenerate critical points of the second class (black lines) switches sign for increasing excitation energy of the intruder configuration $\Delta^{\prime}$.

In the diagram at lower right, we zoom in on the set of critical points of the first type (see eq. (13)) for $\Delta^{\prime}=0$. The inset figure illustrates the behaviour of the energy surface in the $\beta$-direction. In the left triangular region, two minima in the $\beta$-direction exist for $\gamma=\pi / 3$ while in the right triangular region, the two minima in $\beta$ appear for $\gamma=0$. These minima in $\beta$ are very shallow local minima or saddle points, hence we will not discuss them any further.

\subsubsection{SU(3)-SU $(3)$ mixing $\left(\chi_{1}=-\sqrt{7} / 2\right.$ and $\left.\chi_{2}=\sqrt{7} / 2\right)$}

In Fig. 7, we display the phase diagram for $S U(3)-\overline{S U}(3)$ mixing for different values of the scaled excitation energy $\Delta^{\prime}$ of the intruder configuration. Here again, we stress the significant variation in the scale of the $\left|\sigma^{\prime}\right|$ and the $\omega^{\prime}$ axes. Degenerate critical points of the first class (13) and of the second class (14) are shown in blue and black, respectively. For a fixed $\omega^{\prime}$ and increasing $\left|\sigma^{\prime}\right|$, the energy surface changes in a similar way as for $S U(3)-\hat{Q}\left(\chi_{2}\right) \cdot \hat{Q}\left(\chi_{2}\right)$ with $\chi_{2}=\sqrt{7} / 16$ (see previous section), evolving from a prolate minimum, over a region with shape coexistence of a prolate and oblate minimum, to a single oblate minimum. In comparison with Fig 6, the slope of one of the solutions indicated in black has already switched sign, pointing out that the change 
of the slope occurs at negative values of $\Delta^{\prime}$. The IBM parameter $\Delta^{\prime}$ may in principle become negative when the energy needed to excite two particles across the shell gap becomes smaller than the combined pairing energy and the monopole correction. This is for example the case in $\mathrm{Sr}-\mathrm{Zr}$ region [24]. Although calculations for negative $\Delta^{\prime}$ pose no problems, we restrict ourself to positive values in this work.

In the diagram at the lower right of Fig. 7, we zoom in on the set of critical points of the first type (13) (indicated in blue) for $\Delta^{\prime}=0$. The inset figure illustrates the behaviour of the energy surface in the $\beta$-direction. In the left triangular region, two minima in the $\beta$-direction exist for $\gamma=\pi / 3$ while in the right triangular region, the two minima in $\beta$ appear at $\gamma=0$. In comparison to the case of $S U(3)-\hat{Q}\left(\chi_{2}\right) \cdot \hat{Q}\left(\chi_{2}\right)$ with $\chi_{2}=\sqrt{7} / 16$ (see Fig. 6), the minima are more pronounced. However, since we cannot draw general conclusions on the $\gamma$-behaviour of the extrema in the $\beta$-direction for general values of $\Delta^{\prime}$, we only conclude that this $\gamma$-behaviour has to be studied for each value of $\Delta^{\prime}$ separately.

\subsection{Phase diagrams for $O(6)-O(6)$ mixing $\left(\chi_{1}=\chi_{2}=0\right)$}

In case of $O(6)-O(6)$ mixing, all $\gamma$-dependence disappears in the energy surface. Consequently, the phase diagram is determined by the first class of solutions to the criticality conditions which indicates changes of the number of extrema in $\beta$. The phase diagram is very alike to the diagrams in case of prolate-prolate mixing, discussed in Sect. 5.1.1-5.1.3. A triangular region of shape coexistence between two shallow deformed $\gamma$-independent minima is found while outside this area, the energy surface exhibits a single deformed $\gamma$ independent minimum. Similar to the case of $S U(3)-S U(3)$ mixing (see Sect. 5.1.1), the ratio $\beta_{N} / \beta_{N+2}$ converges to one, although a little slower, and no shape coexistence is found when this ratio becomes approximately one.

\section{Conclusions}

In the present work, we have studied the geometry associated with mixing between two deformed configurations, i.e. $\hat{Q}\left(\chi_{1}\right) \cdot \hat{Q}\left(\chi_{1}\right)-\hat{Q}\left(\chi_{2}\right) \cdot \hat{Q}\left(\chi_{2}\right)$ mixing. Mixing between the prolate deformed $S U(3)$ limit $(\chi=-\sqrt{7} / 2)$, the oblate deformed $\overline{S U}(3)$ limit $(\chi=\sqrt{7} / 2)$ and the $\gamma$-independent $O(6)$ limit are contained within this approach. In contrast to the case of $U(5)-\hat{Q}(\chi) \cdot \hat{Q}(\chi)$ mixing, only restricted information can be derived analytically due to the complexity of the energy surface under study. It is, however, possible to organise the solutions to the criticality conditions into two classes. A first class 
indicates where the number of extrema in $\beta$ changes, while a second class announces the transition from a single minimum in $\gamma$ to shape coexistence between a prolate and an oblate minimum. It can be shown that this second class of solutions can only arise if $\chi_{1}$ and $\chi_{2}$ have an opposite sign. Furthermore, the existence of triaxial phases is excluded. As it was not possible to obtain an analytical solution to the criticality conditions, all phase diagrams were constructed numerically.

Various phase diagrams for the selected case of $S U(3)-\hat{Q}\left(\chi_{2}\right) \cdot \hat{Q}\left(\chi_{2}\right)$, with $\chi_{2}$ varying from $-\sqrt{7} / 2$ to $\sqrt{7} / 2$ have been constructed. For prolate intruder configurations, the phase diagram is determined by degenerate critical points of the first class. We find a relatively small region of shape coexistence between two rather shallow prolate deformed minima, provided the minima of the unperturbed regular and the intruder configurations occur at sufficiently different deformations. As the absolute value of $\chi_{2}$ decreases and eventually becomes zero in the $O(6)$ limit, one of the minima in the region with shape coexistence becomes softer in the $\gamma$-direction. Outside the region of shape coexistence, the energy surface exhibits a single deformed minimum. When $\chi_{2}$ becomes positive, indicating an oblate deformation of the intruder configuration, a second class of solutions appears in the phase diagram. The phase diagram now contains regions where the energy surface has a prolate or an oblate deformed minimum and a large region of shape coexistence between pronounced and clearly separated prolate and oblate minima. The first class of degenerate critical points delineates two small regions of shape coexistence in $\beta$. Because the $\gamma$-behaviour at the created additional extremum in $\beta$ is not a priori known, we have not discussed them any further. Finally, we have calculated the phase diagram for $O(6)-O(6)$ mixing. The first class of degenerate critical points gives rise to a small region of shape coexistence between two $\gamma$-independent minima. Again, the minima are shallow.

\section{Acknowledgements}

We thank the Institute for Nuclear Theory at the University of Washington for its hospitality and the Department of Energy (DOE) for partial support during the completion of this work. Financial support from the "FWO Vlaanderen" (V.H. and K.H), the University of Ghent (S.D.B. and K.H.) and the Interuniversity Attraction Pole (IUAP) under projects P5/07 and P6/23 is gratefully acknowledged. V.H. and S.D.B. also received financial support from the European Union under contract No. 2000-00084. 


\section{A Derivatives of $E_{-}$to $\gamma$}

The first derivative with respect to $\gamma$ of the energy surface (7) can be written as

$$
\frac{\partial E_{-}}{\partial \gamma}=\frac{3 \beta^{3} \sin (3 \gamma)}{\left(1+\beta^{2}\right)^{2}} F\left(\beta, \gamma ;\left|\sigma^{\prime}\right|, N, \Delta^{\prime}, \chi_{1}, \chi_{2}, \omega^{\prime}\right)
$$

with

$$
\begin{aligned}
& F\left(\beta, \gamma ;\left|\sigma^{\prime}\right|, N, \Delta^{\prime}, \chi_{1}, \chi_{2}, \omega^{\prime}\right)= \\
& \frac{\left.b_{3}\left[b_{1} \beta^{4}+b_{2} \beta^{2}+b_{3} \beta^{3} \cos (3 \gamma)+b_{4}\right)\right]}{\left\{\left[b_{1} \beta^{4}+b_{2} \beta^{2}+b_{3} \beta^{3} \cos (3 \gamma)+b_{4}\right]^{2}+\omega^{\prime 2}\left(1+\beta^{2}\right)^{4}\right\}^{1 / 2}}-a_{3} .
\end{aligned}
$$

Hence, the second derivative with respect to $\gamma$ becomes

$$
\frac{\partial^{2} E_{-}}{\partial \gamma^{2}}=\frac{9 \beta^{3} \cos (3 \gamma)}{\left(1+\beta^{2}\right)^{2}} F+\frac{3 \beta^{3} \sin (3 \gamma)}{\left(1+\beta^{2}\right)^{2}} \frac{\partial F}{\partial \gamma}
$$

with

$$
\frac{\partial F}{\partial \gamma}=\frac{-3 b_{3}^{2} \beta^{3} \sin (3 \gamma) \omega^{\prime 2}\left(1+\beta^{2}\right)^{4}}{\left\{\left[b_{1} \beta^{4}+b_{2} \beta^{2}+b_{3} \beta^{3} \cos (3 \gamma)+b_{4}\right]^{2}+\omega^{\prime 2}\left(1+\beta^{2}\right)^{4}\right\}^{3 / 2}} .
$$

\section{B Analysis of the extremum in case of prolate-prolate mixing}

In this appendix, we calculate the $\gamma$-behaviour of the minima in $\beta$ in case of mixing between a prolate regular and a prolate intruder configuration.

If both $\chi_{1}$ and $\chi_{2}$ are chosen negative in (7) and if take $\gamma=0$, the minima in the $\beta$-direction with $\beta>0$ (prolate) correspond to a minimum in $\gamma$, as will be shown further on. Similarly, the minima in the $\beta$-direction with $\beta<0$ (oblate, since $E_{-}\left(\beta_{0}, \gamma=\pi / 3\right)=E_{-}\left(-\beta_{0}, \gamma=0\right)$ ) correspond to a maximum in $\gamma$ such that these extrema are saddle-points. This implies that we may omit solutions to the criticality conditions of the first class with $\beta<0$ in Sect. 5.1.15.1 .2 as these describe only a coexistence between shallow oblate saddle points.

It follows from eq. (11) that, along the $\gamma=0$ line, the energy surface exhibits an extremum in the $\gamma$ direction for every $\beta$. The second derivative $\partial^{2} E_{-} /\left.\partial \gamma^{2}\right|_{\gamma=0}$ determines whether this extremum is a minimum or a maxi- 
mum in $\gamma$. From (A.3), we find

$$
\begin{aligned}
\left.\frac{\partial^{2} E_{-}}{\partial \gamma^{2}}\right|_{\gamma=0}=\frac{9 \beta_{0}^{3}}{\left(1+\beta_{0}^{2}\right)^{2}}( & -a_{3} \\
& \left.+\frac{b_{3}\left(b_{1} \beta_{0}^{4}+b_{2} \beta_{0}^{2}+b_{3} \beta_{0}^{3}+b_{4}\right)}{\sqrt{\left(b_{1} \beta_{0}^{4}+b_{2} \beta_{0}^{2}+b_{3} \beta_{0}^{3}+b_{4}\right)^{2}+\omega^{\prime 2}\left(1+\beta_{0}^{2}\right)^{4}}}\right)
\end{aligned}
$$

where $\beta_{0}$ denotes the deformation of the minimum in the $\beta$-direction. From (8), we derive that

$$
\begin{aligned}
& a_{3}+b_{3}=\frac{8}{7} \sqrt{14} N(N-1) \chi_{1}, \\
& a_{3}-b_{3}=\frac{8}{7} \sqrt{14}\left|\sigma^{\prime}\right|(N+1)(N+2) \chi_{2} .
\end{aligned}
$$

Because both $\chi_{1}$ and $\chi_{2}$ are negative in case of prolate-prolate mixing, this implies that

$$
a_{3}<b_{3}<-a_{3} .
$$

From (8), we also learn that $a_{3}<0$ in case of prolate-prolate mixing. If we define

$$
\begin{aligned}
& f_{1}=b_{1} \beta_{0}^{4}+b_{2} \beta_{0}^{2}+b_{3} \beta_{0}^{3}+b_{4} \\
& f_{2}=\sqrt{\left(b_{1} \beta_{0}^{4}+b_{2} \beta_{0}^{2}+b_{3} \beta_{0}^{3}+b 4\right)^{2}+\omega^{\prime 2}\left(1+\beta_{0}^{2}\right)^{4}}
\end{aligned}
$$

then $\left|f_{1}\right| \leq f_{2}$. If $f_{1}<0$, then we may derive from $b_{3}<-a_{3}$ that $f_{1} b_{3}>-f_{1} a_{3}$ or $\left|f_{1}\right| a_{3}<f_{1} b_{3}$. Since $\left|f_{1}\right| \leq f_{2}$ and $a_{3}<0, f_{2} a_{3} \leq\left|f_{1}\right| a_{3}$. Hence, for $f_{1}<0$, $f_{2} a_{3}<f_{1} b_{3}$. Similar, when $f_{1}>0, f_{1} a_{3}<f_{1} b_{3}$ or $f_{2} a_{3}<f_{1} b_{3}$. Consequently, the factor

$$
\left(-a_{3}+\frac{b_{3}\left(b_{1} \beta_{0}^{4}+b_{2} \beta_{0}^{2}+b_{3} \beta_{0}^{3}+b_{4}\right)}{\sqrt{\left(b_{1} \beta_{0}^{4}+b_{2} \beta_{0}^{2}+b_{3} \beta_{0}^{3}+b_{4}\right)^{2}+\omega^{\prime 2}\left(1+\beta_{0}^{2}\right)^{4}}}\right)>0
$$

and the sign of $\beta_{0}$ determines the sign of $\partial^{2} E_{-} /\left.\partial \gamma^{2}\right|_{\gamma=0}$. For $\beta_{0}<0$, the minimum in the $\beta$-direction is a saddle-point in the $(\beta, \gamma)$-plane. For $\beta_{0}>0$, the minimum in the $\beta$-direction is a minimum in the $(\beta, \gamma)$-plane. If $f_{2}=0$, then $f_{1}=0$ and

$$
\left.\frac{\partial^{2} E_{-}}{\partial \gamma^{2}}\right|_{\gamma=0}=-\frac{9 a_{3} \beta_{0}^{3}}{\left(1+\beta_{0}^{2}\right)^{2}},
$$

and since $a_{3}<0$, the sign of $\beta_{0}$ again determines whether the minimum in the $\beta$-direction is a minimum or a saddle point in the $(\beta, \gamma)$ plane. In case of oblate-oblate mixing the analysis proceeds in a similar way. 


\section{References}

[1] F. Iachello and A. Arima, The interacting boson model (Cambridge University Press, Cambridghe, 1987).

[2] P. Cejnar and J. Jolie, Prog. Part. Nucl. Phys. (2008) In Press, Corrected Proof.

[3] J.N. Ginocchio and M.W. Kirson, Phys. Rev. Lett. 44 (1980) 1744.

[4] A.E.L. Dieperink, O. Scholten and F. Iachello, Phys. Rev. Lett. 44 (1980) 1747.

[5] A. Bohr and B. Mottelson, Phys. Scripta 22 (1980) 468.

[6] D.H. Feng, R. Gilmore and S.R. Deans, Phys. Rev. C 23 (1981) 1254.

[7] E. López-Moreno and O. Castaños, Phys. Rev. C 54 (1996) 2374.

[8] J. Jolie et al., Phys. Rev. Lett. 89 (2002) 182502.

[9] J. Jolie et al., Phys. Rev. Lett. 87 (2001) 162501.

[10] F. Iachello and N.V. Zamfir, Phys. Rev. Lett. 92 (2004) 212501.

[11] D.J. Rowe, Nucl. Phys. A 745 (2004) 47.

[12] G. Rosensteel and D.J. Rowe, Nucl. Phys. A 759 (2005) 92.

[13] A. Leviatan, Phys. Rev. C 74 (2006) 051301(R).

[14] R.F. Casten, Nat. Phys. 2 (2006) 811.

[15] P.D. Duval and B.R. Barrett, Phys. Lett. B 100 (1981) 223.

[16] P.D. Duval and B.R. Barrett, Nucl. Phys. A 376 (1982) 213.

[17] K. Heyde et al., Phys. Rep. 102 (1983) 291.

[18] J.L. Wood et al., Phys. Rep. 215 (1992) 101.

[19] A. Frank, P. Van Isacker and C.E. Vargas, Phys. Rev. C 69 (2004) 034323.

[20] V. Hellemans et al., Nucl. Phys. A 789 (2007) 164.

[21] I.O. Morales et al., Phys. Rev. C 78 (2008) 024303.

[22] A. Frank and P. Van Isacker, Algebraic methods in molecular and nuclear structure physics (John Wiley and Sons, Inc., New York, 1994).

[23] D.D. Warner and R.F. Casten, Phys. Rev. C 28 (1983) 1798.

[24] K. Heyde et al., Nucl. Phys. A 466 (1987) 189.

[25] R. Gilmore, Catastrophe theory for scientists and engineers (Wiley, New York, 1981).

[26] M.A. Caprio and F. Iachello, Ann. of Phys. 318 (2005) 454.

[27] P. Van Isacker and J.Q. Chen, Phys. Rev. C 24 (1981) 684.

[28] K. Heyde et al., Nucl. Phys. A 398 (1983) 235. 

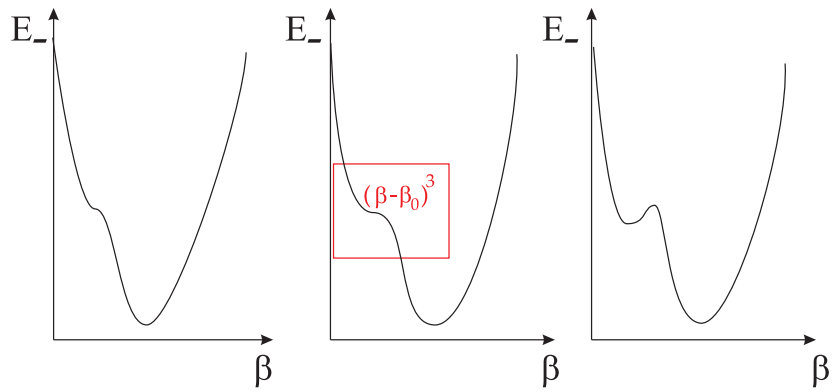

Fig. 1. Schematic illustration of the transition from one deformed minimum to two deformed minima in the $\beta$-direction. The $\beta^{3}$-behaviour at the degenerate critical point is indicated with a red box.
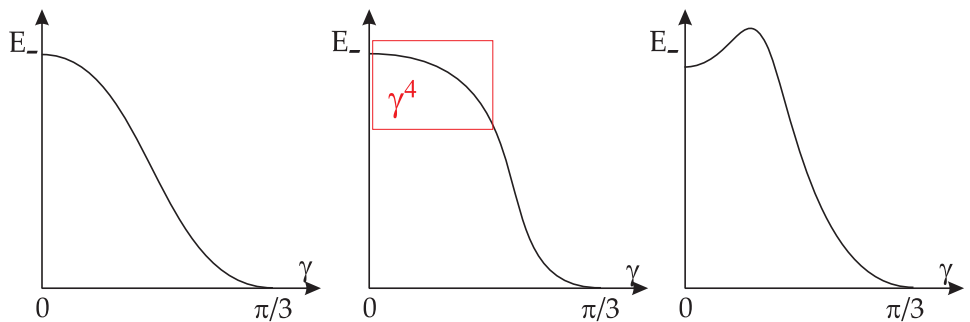

Fig. 2. Schematic illustration of the transition from one minimum to two minima in the $\gamma$-direction $\left(\beta=\beta_{0}\right)$. The $\gamma^{4}$ behaviour at the degenerate critical point is indicated with a red box. 

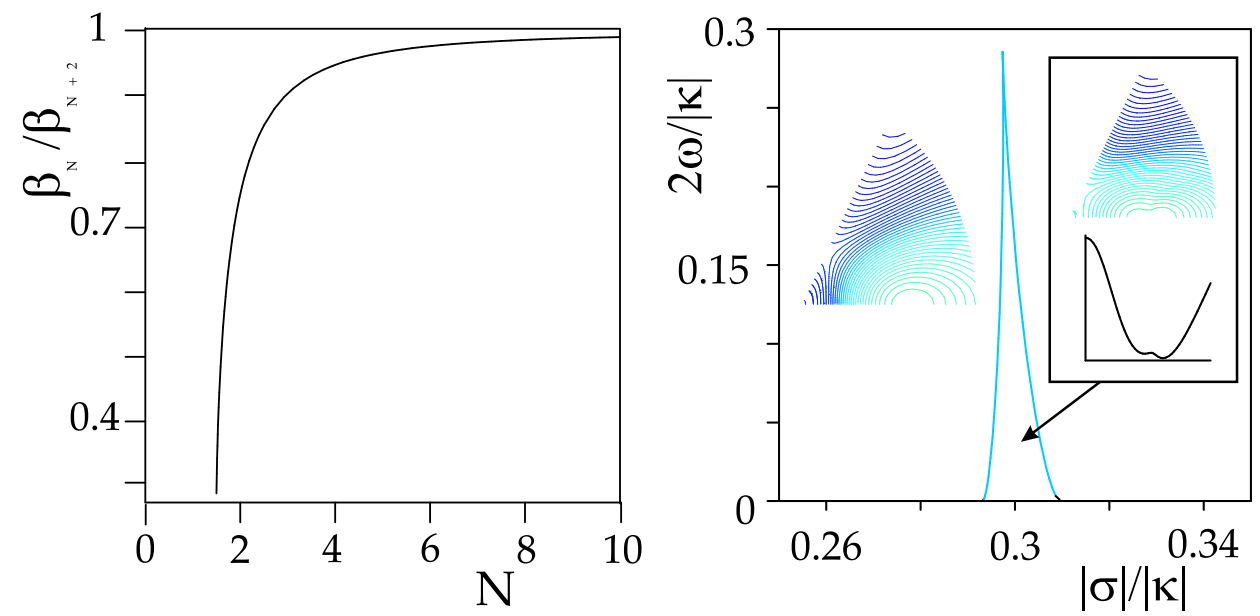

Fig. 3. $S U(3)-S U(3)\left(\chi_{1}=\chi_{2}=-\sqrt{7} / 2\right)$. Panel (a) displays the ratio of the deformation at the minimum of the energy surface associated with the regular configuration to the deformation at the minimum of the energy surface associated with the intruder configuration. Panel (b) shows the phase diagram for $\Delta /|\kappa|=0$ and boson number $N=2$. The phase diagram is shown as a function of the ratio of the strengths of the quadrupole interaction $|\sigma| /|\kappa|$ and the scaled strength of the mixing between the two configurations $2 \omega /|\kappa|$. The inset contour plots illustrate the generic shape of the energy surface in the $(\beta, \gamma)$ plane in each of the zones of the parameter space. Minima are depicted in light blue and the blue grows darker as the energy rises. The one-dimensional inset plot shows the evolution of the energy surface in $\beta$ for $\gamma=0$.
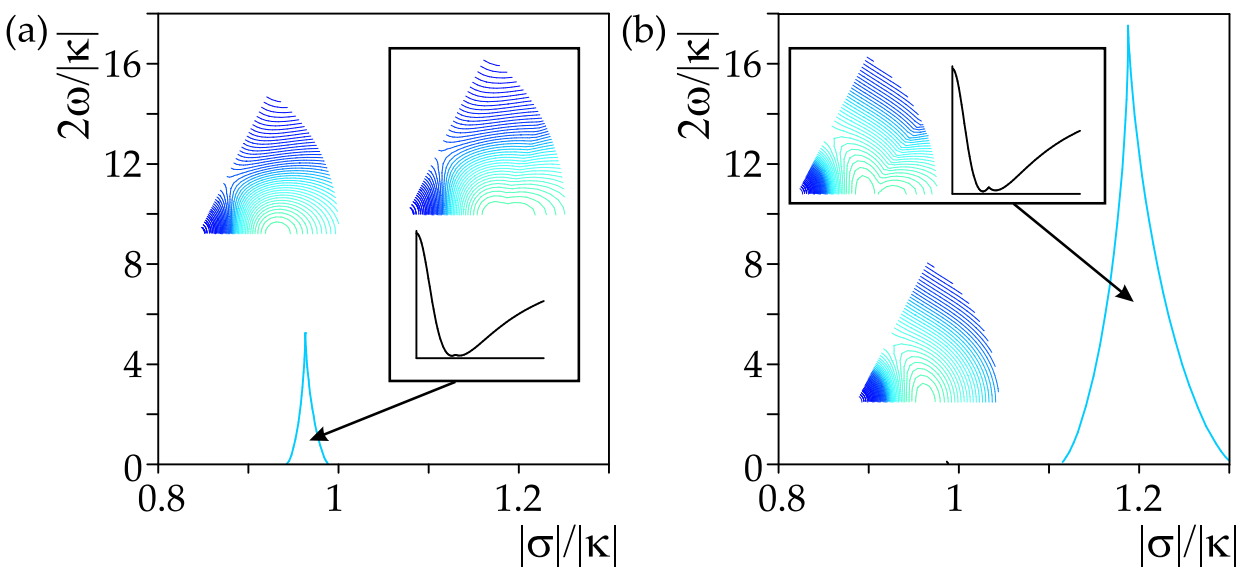

Fig. 4. Phase diagrams for $S U(3)-\hat{Q}\left(\chi_{2}\right) \cdot \hat{Q}\left(\chi_{2}\right)$ : in panel (a) $\chi_{2}=-\sqrt{7} / 4$ and in panel (b) $\chi_{2}=-\sqrt{7} / 16$. Both panels display the phase diagram for $\Delta /|\kappa|=0$ and $N=10$ as a function of the ratio of the strengths of the quadrupole interaction $|\sigma| /|\kappa|$ and the scaled strength of the mixing between the two configurations $2 \omega /|\kappa|$. The inset contour plots illustrate the generic shape of the energy surface in the $(\beta, \gamma)$ plane in each of the zones of the parameter space. The one-dimensional inset plots show the evolution of the energy surface in $\beta$ for $\gamma=0$. In the contour plots, minima are depicted in light blue and the blue grows darker as the energy rises. 

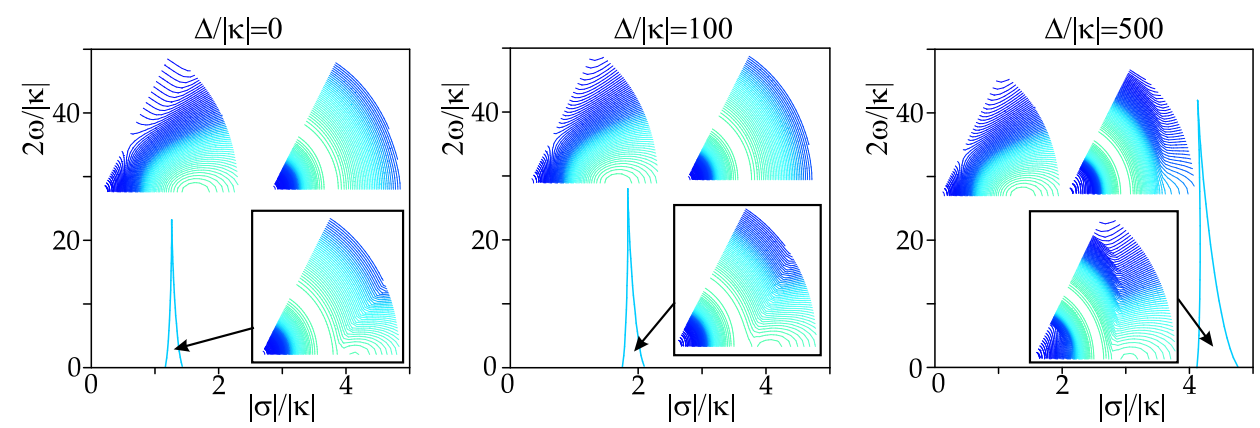

Fig. 5. Phase diagrams for $S U(3)-O(6)$ mixing $\left(\chi_{1}=-\sqrt{7} / 2\right.$ and $\left.\chi_{2}=0\right)$ for different values of the scaled excitation energy of the intruder states $\Delta /|\kappa|$ and for $N=10$. The phase diagrams are shown as a function of the ratio of the strengths of the quadrupole interaction $|\sigma| /|\kappa|$ and the scaled strength of the mixing between the two configurations $2 \omega /|\kappa|$. The inset contour plots illustrate the generic shape of the energy surface in the $(\beta, \gamma)$ plane in each of the zones of the parameter space. For the meaning of the two inset plots outside the triangular region, we refer to the discussion in the text. Minima are depicted in light blue and the blue grows darker as the energy rises. 

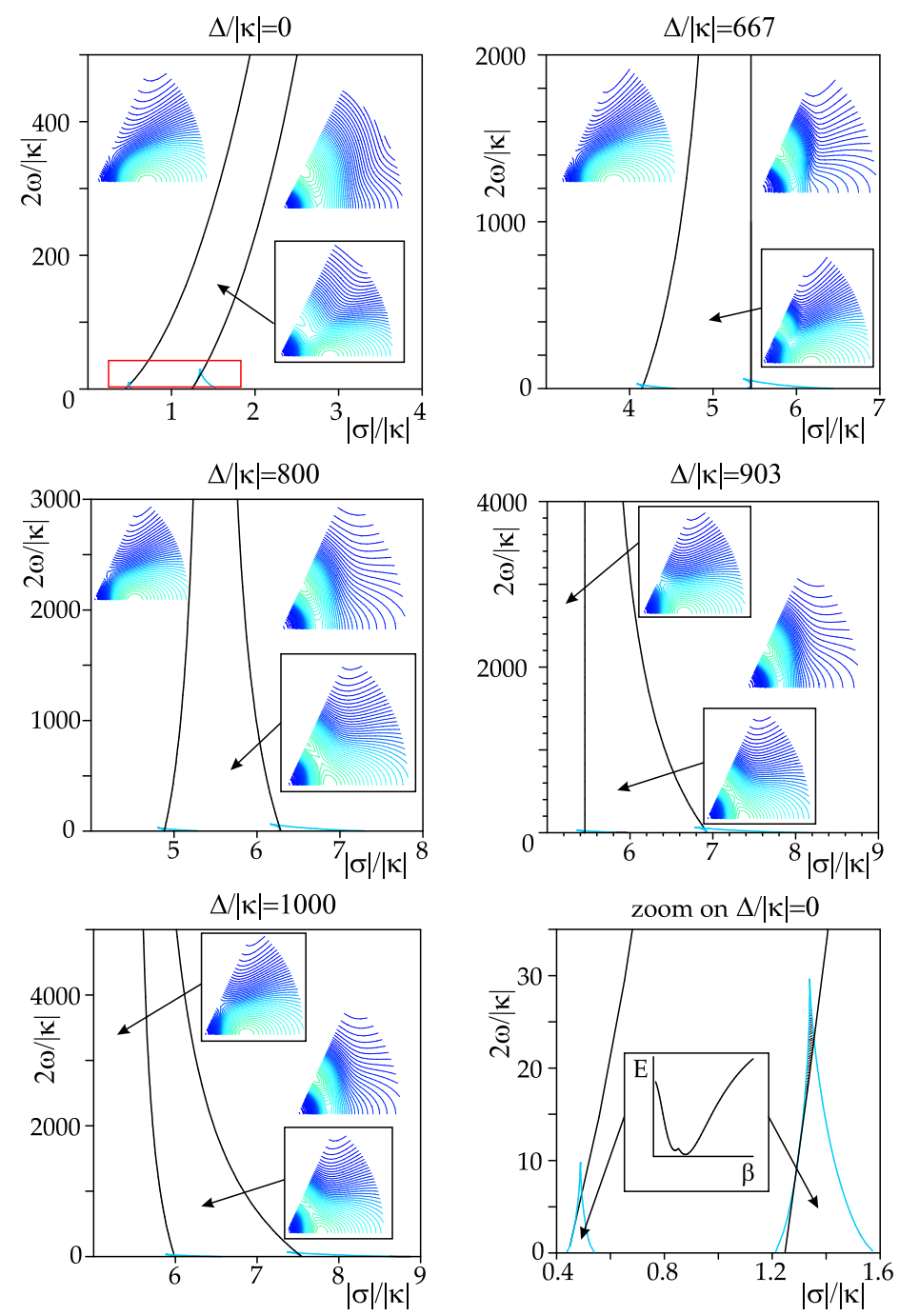

Fig. 6. Phase diagrams for $S U(3)-\hat{Q}\left(\chi_{2}\right) \hat{Q}\left(\chi_{2}\right)$ mixing $\left(\chi_{1}=-\sqrt{7} / 2\right.$ and $\left.\chi_{2}=\sqrt{7} / 16\right)$ for different values of $\Delta /|\kappa|$ and for $N=10$. The phase diagrams are shown as a function of the ratio of the strengths of the quadrupole interaction $|\sigma| /|\kappa|$ and the scaled strength of the mixing between the two configurations $2 \omega /|\kappa|$. Degenerate critical points of the first class (13) and of the second class (14) are shown in blue and black respectively. The inset contour plots illustrate generic shape of the energy surface in the $(\beta, \gamma)$ plane in each of the zones of the parameter space. Minima are depicted in light blue and the blue grows darker as the energy rises. In the diagram at the lower right, a zoom on the degenerate critical points of the first class for $\Delta /|\kappa|=0$ is provided. We stress the changing scale of the $|\sigma| /|\kappa|$ and the $2 \omega /|\kappa|$ axes in the different phase diagrams. 

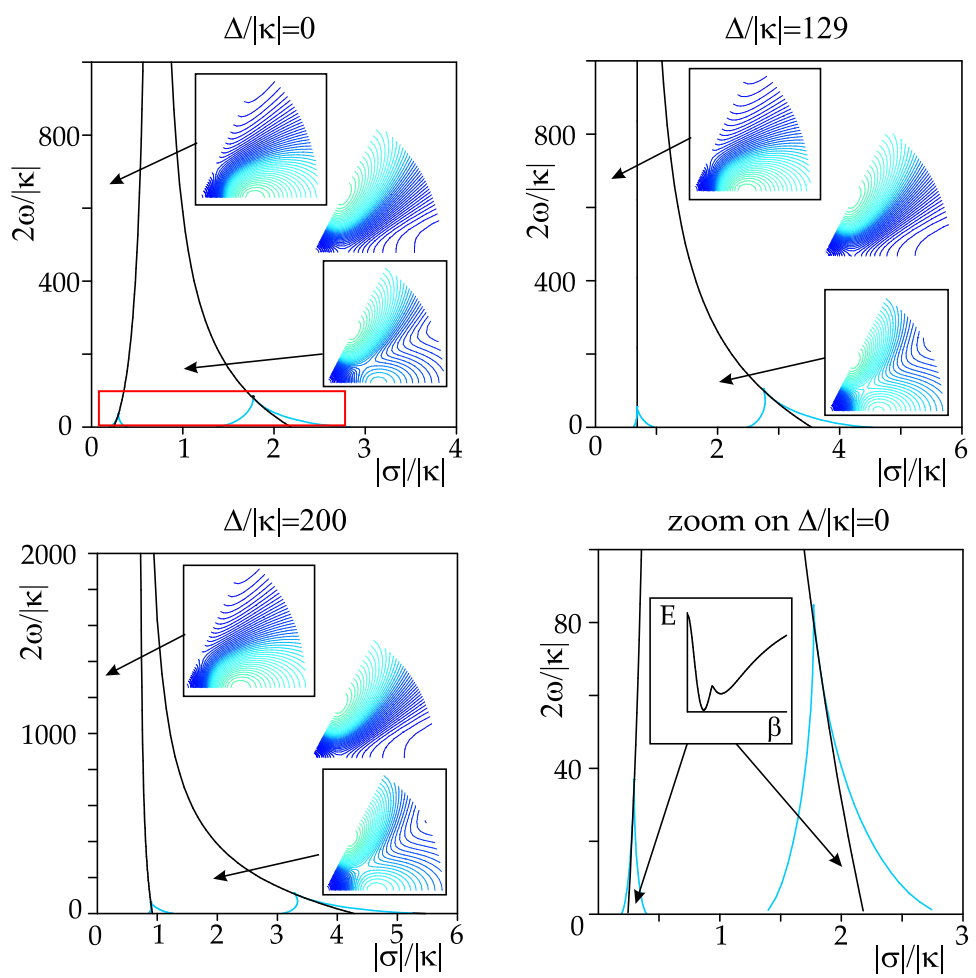

Fig. 7. Phase diagrams for $S U(3)-\overline{S U}(3)$ mixing $\left(\chi_{1}=-\sqrt{7} / 2\right.$ and $\left.\chi_{2}=\sqrt{7} / 2\right)$ for different values of $\Delta /|\kappa|$ and for $N=10$. The phase diagrams are shown as a function of the ratio of the strengths of the quadrupole interaction $|\sigma| /|\kappa|$ and the scaled strength of the mixing between the two configurations $2 \omega /|\kappa|$. Degenerate critical points of the first class (13) and of the second class (14) are shown in blue and black respectively. The inset contour plots illustrate generic shape of the energy surface in the $(\beta, \gamma)$ plane in each of the zones of the parameter space. Minima are depicted in light blue and the blue grows darker as the energy rises. In the diagram at the lower right, a zoom on the degenerate critical points of the first class for $\Delta /|\kappa|=0$ is provided. 